\title{
Flammulina as a model system for fungal graviresponses
}

\author{
Volker D. Kern ${ }^{1 *}$, Kurt Mendgen ${ }^{2}$, Bertold Hock ${ }^{1}$ \\ ${ }^{1}$ Lehrstuhl für Botanik, Technische Universität München at Freising-Weihenstephan, D-85350 Freising, Germany \\ ${ }^{2}$ Lehrstuhl für Phytopathologie, Universität Konstanz, D-78434 Konstanz, Germany
}

Received: 27 June 1996 / Accepted: 20 February 1997

\begin{abstract}
Gravitropic bending of fruiting bodies of Flammulina velutipes (Curtis) Karst. is based on the differential growth of the transition zone between stem and cap. Reorientation becomes visible as early as $2 \mathrm{~h}$ after displacing the fruiting body from the vertical to the horizontal position. It is preceded by a preferential accumulation of microvesicles within the hyphae on the lower side of the transition zone and related to an increase in the vacuolar compartment required for hyphal extension. A model made of a bundle of interconnected balloons is used to demonstrate that a differential volume increase at one flank is sufficient to bend the entire structure in the opposite direction. Gravitropic raising of intact stems or segments derived from the transition zone requires positional information which can be accomplished by three major, coordinated events: (i) gravisensing by the individual hyphae within the transition zone, (ii) unidirectional signalling by means of a soluble growth factor creating a vertical concentration gradient, and (iii) translation of the concentration signal into elongation growth.
\end{abstract}

Key words: Basidiomycete - Gravitropism - Flammulina - Microvesicle - Positional information

\section{Introduction}

In the last century, the gravireaction of basidiomycete fruiting bodies drew the attention of biologists such as Schmitz (1842), Hofmeister (1860) and Sachs (1865, 1879). Major progress has been achieved during the last

\footnotetext{
* Present address: Department of Plant Biology, Ohio State University, 1735 Neil Ave., Columbus, OH 48210, USA

Abbreviation: TEM $=$ transmission electron microscopy

Correspondence to: B. Hock; Fax: 49(8161)714403;

E-mail: hock@pollux.edv.agrar.tu-muenchen.de
}

decade with respect to graviresponses in fungi. This has been extensively reviewed by Moore et al. (1996). However, the mechanism of graviperception in fungi is far from being settled, yet. The classical concept of dynamic gravistimulation in plants (cf. Sievers et al. 1991) is based on the ideas of Haberlandt (1900) and Němec (1900) who related the sedimentation of amyloplasts to gravity sensing. Since fungi are devoid of plastids, other sensing devices have been discussed in the past.

Dennison (1961) and Dennison and Shropshire (1984) attributed the gravitropic response of Phycomyces sporangiophores to the upward floating of vacuoles. Protoplasmic asymmetry was assumed to cause the differential growth responsible for negative gravitropism. It was postulated that the thicker layer of protoplasm below the vacuole generates more wall growth on the lower side. As is demonstrated below, this mechanism can not be applied to the gravitropic bending of a fruiting-body stem, which is composed of many individual hyphae firmly attached to each other, especially at the periphery of the stem.

Mechanical forces acting on the whole fruiting body have been excluded as a means of graviperception (Greening et al. 1993). Monzer and Haindl (1994) and Monzer (1995) extended the concept of the cytoskeleton as an essential element in gravisensing (Sievers et al. 1991) to fungal stems. It was shown for Flammulina that the nuclei are embedded in a web of F-actin. Nuclear displacement within the actin filaments could generate a signal large enough to be detected by the hypothetical sensing mechanism. True sedimentation of the nuclei has not been observed so far (Moore 1991; Monzer and Haindl 1994).

Gravitropic bending is understood to be a prerequisite for optimal spore dispersal. Buller (1909) has shown that a deviation of the lamellae by only $5^{\circ}$ from the vertical position leads to a $50 \%$ loss of total spore dispersal. Definite proof for the directional growth of fruiting bodies as a response to a unidirectional gravity vector has been given by an experiment in weightlessness during the second German spacelab mission D-2 in 1993. 

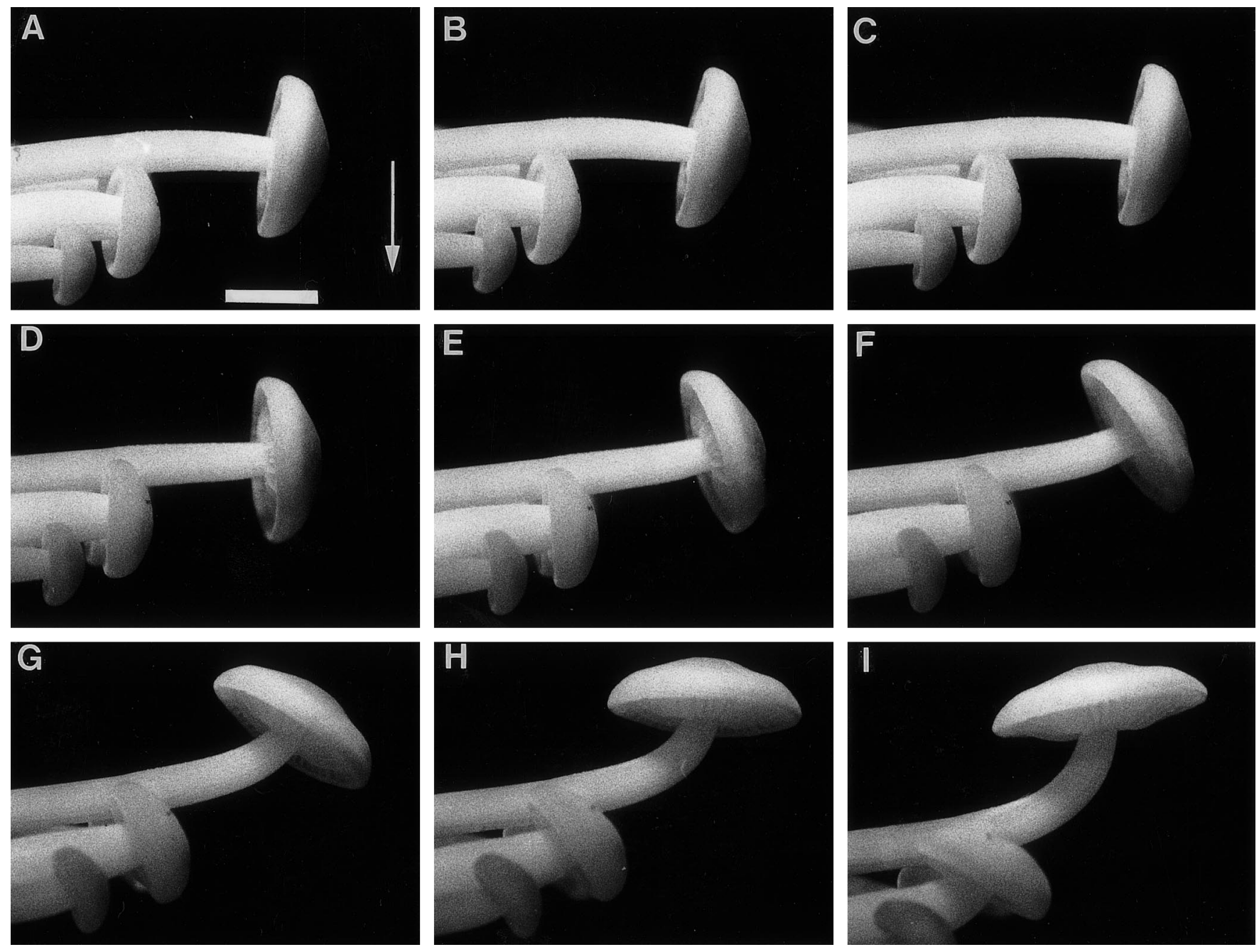

Fig. 1A-I. Graviresponse of Flammulina velutipes (Curtis) Karst fruiting bodies after reorientation to the horizontal position at time $0 \mathrm{~h}$ (A), $0.5 \mathrm{~h}$ (B), 1 h (C), 2 h (D), 3 h (E), 4 h (F), 7 h (G), 10 h (H), $16 \mathrm{~h}$ (I). Arrow, gravitational vector. The photographs were taken in low-intensity red light to avoid phototropic effects. Hybrid strains of Flammulina were derived from crossing a standard strain (optimal fruiting temperature at $13{ }^{\circ} \mathrm{C}$; obtained by Prof. C. Thielke, Freie Universität, Berlin) with the heat-tolerant strain RMBL6 (fruiting at $22{ }^{\circ} \mathrm{C}$; obtained by Prof. S.A. Fultz, Stanford University, Calif., USA; see Fultz 1988). Cultures were grown as described earlier (Kern and Hock 1994) on wood shavings from willow supplemented with wheat bran and liquid nutrient (malt extract and peptone in aqueous solution, $\mathrm{pH}$ 5.6). Bar $=1 \mathrm{~cm}$

Whereas microgravity-grown fruiting bodies of Flammulina velutipes exhibited random orientation, cultures grown on a $1 \mathrm{~g}$ centrifuge in space developed fruiting bodies which pointed in exactly the opposite direction to the acceleration force (Kern and Hock 1994).

In Flammulina, elongation growth and gravitropic bending are restricted to a 2- to $3-\mathrm{mm}$ apical zone of the stem, the transition zone, where the cap and the stem hyphae interconnect. The gravitropic response is due to growth inhibition on the upper side of the stem. Monzer et al. (1994) demonstrated that even small stem segments carry out gravitropic curvature as long as the transition zone is included. The same holds true if the apical part of the stem is split into small longitudinal segments and then horizontally oriented. All segments exhibit individual gravitropic bending irrespective of the position of their insertion at the stem. Segments attached at the lower side bend upwards equally as strongly as the segments of the upper side. This result is thought to support the view of Hofmeister (1863) who worked with longitudinally split stems of Amanita. He concluded that each of the numerous stem hyphae has the individual capacity to respond to reorientation. However, it is not clear how the individual responses of single hyphae are coordinated in an intact system.

Unfortunately, little is known about hyphal extension in stems, which follows a pattern distinctly different from that of mycelial hyphae. Whereas the latter elongate by tip growth, the former carry out diffuse elongation growth (for review, cf. Wessels 1993); nothing is known about the control of tropic bending in the latter case.

This paper is an attempt to integrate into a new model some new structural observations obtained from bending stipes and earlier physiological work obtained by our group. It relates gravisensing by individual hyphae and unidirectional signalling to the generation 
of positional information required for the coordinated bending reaction in response to a gravitational vector.

\section{Graviresponse in the model system Flammulina}

When a culture of Flammulina velutipes is shifted from the vertical to the horizontal position, gravitropic bending of the fruiting bodies becomes visible within $2 \mathrm{~h}$ and approaches $90^{\circ}$ within about $12 \mathrm{~h}$, followed by an overshoot. Figure 1 A-I shows a photographic recording of an intact culture during the first $16 \mathrm{~h}$. Immediately after the fruiting body is brought to a horizontal position, the cap bends down because of its own weight. Knoll (1909) called this phenomenon "Hutlast". Figure 2 presents the kinetics of gravitropic curvature, and shows a lag phase of approximately $2 \mathrm{~h}$.

Since gravisensing and gravireaction, as well as ordinary extension growth, are restricted to the apical zone of the stem, analysis of gravitropic bending must take into account the structural organization of this region (Fig. 3A,B). An outer zone with small and lessvacuolated hyphae gradually merges into an inner zone with larger and highly vacuolated cells. The strictly parallel arrangement of the hyphae (except for a peripheral layer of cystides) is clearly recognizable in the longitudinal section. This arrangement provides the basis for tissue stresses within the stem, which are easily demonstrated by the classical experiment of splitting the apical part of a vertical stem into several longitudinal strips: as in higher-plant stems (Kraus 1867; Kutschera 1992) the inner tissues extend more than the peripheral ones. Therefore, the strips bend outward (not shown) although entirely different tissue constructs are used by the different systems. This gives a first clue that the relative resistence of the peripheral hyphae to tension must be overcome locally during gravitropic bending.

Continued turgor pressure as the essential component of cell elongation depends on an expansion of the vacuolar compartment. It is obvious that vacuolation of hyphae with dense cytoplasm could provide the driving force for a fast redirection of growth. A transmission-

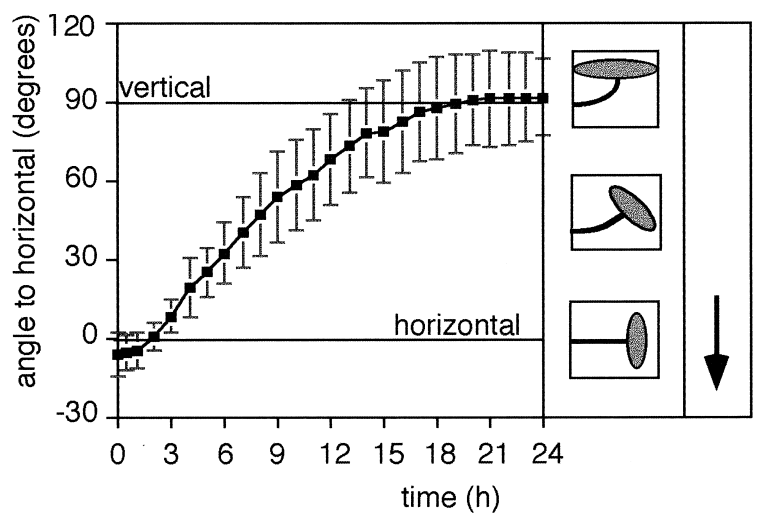

Fig. 2. Kinetics of gravitropic curvature of $F$. velutipes fruiting-body stems after reorientation to the horizontal position. Standard deviations $(n \geq 10)$ are indicated by error bars. Arrow, gravitational vector electron microscopy (TEM) analysis of the transition zone shows that cells undergoing fast vacuolation have electron-lucent regions around their vacuoles. These regions are composed of small, light vesicle-like structures, which are here termed microvesicles.

Figure 4A gives a diagrammatic representation of the distribution of microvesicles across a transverse section of a transition zone from vertically growing fruiting bodies. Representative TEM images are shown for the periphery (Fig. 4B), the adjacent inner zone (Fig. 4C,E) and the center (Fig. 4D,F). The TEM analysis shows a concentric zonation with increasing numbers of microvesicles towards the center, as would be expected for vertical growth. Table 1 presents a quantitative analysis of vesicle areas in cross-sections of stems which had been horizontally oriented for $3 \mathrm{~h}$. The data show that vesicles in hyphae of the upper flank contribute to $6 \%$ of the hyphal area vs. $37 \%$ in hyphae of the lower flank.

The details of the vesiculation process were studied in high-pressure-frozen and freeze-substituted hyphae (Fig. 5A,B) to exclude the possibility that microvesicles are artifacts of chemical fixation. Microvesicles appeared to originate in the cytosol (Fig. 5A). The irregular shape of the tonoplast suggests a fusion of these vesicles with the vacuole (Fig. 5B). Contact with other membrane systems was not found. A correlation between accumulation of microvesicles and extension growth of hyphae becomes obvious during gravitropic bending when elongation preferentially takes place on the lower side of the stem. The vesiculation is seen on the lower flank, well before any growth response can be registered macroscopically. Figure 6 summarizes schematically the distribution of microvesiculation across a transverse section. A distinct shift can be recognized as early as $30 \mathrm{~min}$ after displacing the fruiting bodies to the horizontal position. Representative micrographs from the periphery of the upper and lower side are shown in Fig. 7A,B, demonstrating the conspicuous ultrastructural difference in vesiculation.

The original pattern of concentric arrangement of microvesicle distribution becomes restored when the bending reaction approaches its final position. This becomes evident by comparing the transverse sections (a) and (b) in Fig. 6 at 9 h after displacement when the transition zone has already moved from its earlier position (b) to (a).

\section{Discussion}

Gravitropic bending of the fruiting body stem is preceded by a conspicuous microvesiculation on the lower side of the transition zone. The subcellular sequence of events leading to differential growth is hypothesized to consist of microvesicle fusion with the vacuoles, enlargement of the vacuome and increase in turgor pressure resulting in hyphal extension. Although many details remain open at this time, it is probable that a key event is the signal which leads to microvesicle formation in response to a positional shift with respect to the gravitational vector. 

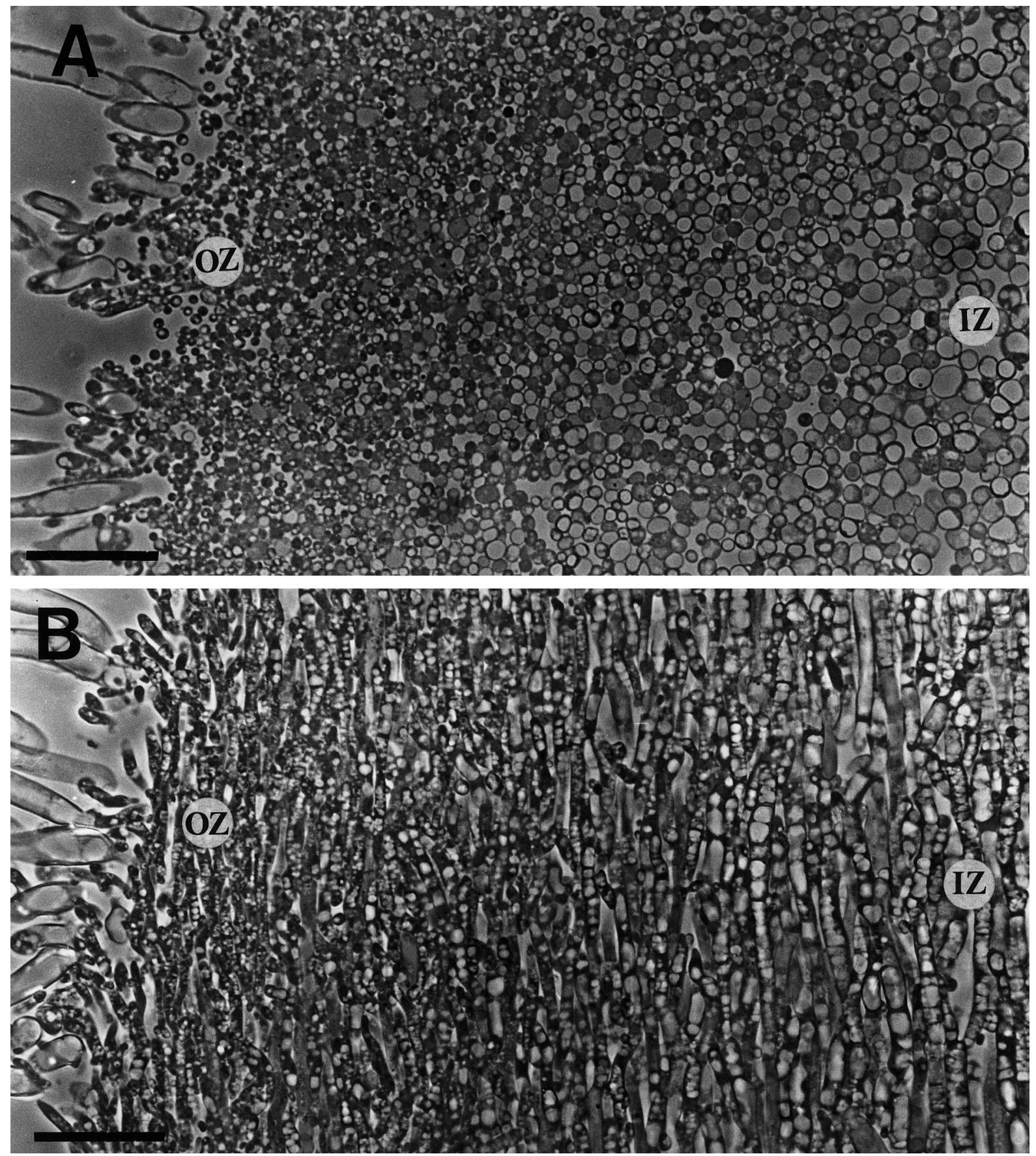

Fig. 3A,B. Light micrographs of the anatomy of the transition zone of the stem of a $F$. velutipes fruiting body. A Transverse section; B longitudinal section. $O Z$, outer zone, $I Z$, inner zone. Fruiting bodies were fixed in $3 \%(\mathrm{v} / \mathrm{v})$ glutardialdehyde in $75 \mathrm{mM}$ potassium phosphate buffer $\left(\mathrm{pH} 7.2\right.$ ) for $2 \mathrm{~h}$, postfixed with $2 \% \mathrm{OsO}_{4}$ for $1 \mathrm{~h}$ and stained with $0.5 \%(\mathrm{w} / \mathrm{v})$ uranylacetate. Dehydration was carried out with increasing ethanol concentrations, followed by a treatment with propylene oxide, and incubation and polymerization for $8 \mathrm{~h}$ at $70{ }^{\circ} \mathrm{C}$ in ERL (Spurr 1969). Semi-thin sections $(2 \mu \mathrm{m})$ were mounted on microscope slides in Entellan (Merck, Darmstadt, Germany). Specimens were analyzed and photographed with a Zeiss Axioplan microscope (Carl Zeiss, Oberkochen, Germany). Bars $=50 \mu \mathrm{m}$
Differential growth of the lower-flank hyphae as the cause of bending rules out alternative models of the gravitropic response. The compatibility with mechanical requirements is illustrated by models where the stem is represented by a bundle of inflatable balloons (Fig. 8A-H). Comparable approaches have been used previously by Aylor et al. (1973) to model the bending of guard cells. A single balloon can easily be bent (Fig. 8B) if one side is reinforced before inflation by adhesive tape running from one end to the other (Fig. 8A). If this principle is applied to the cellular level, a single, horizontally oriented hypha would rise upwards if its upper cell wall were to yield less to turgor increase than its lower cell wall. The same would happen to hyphal 

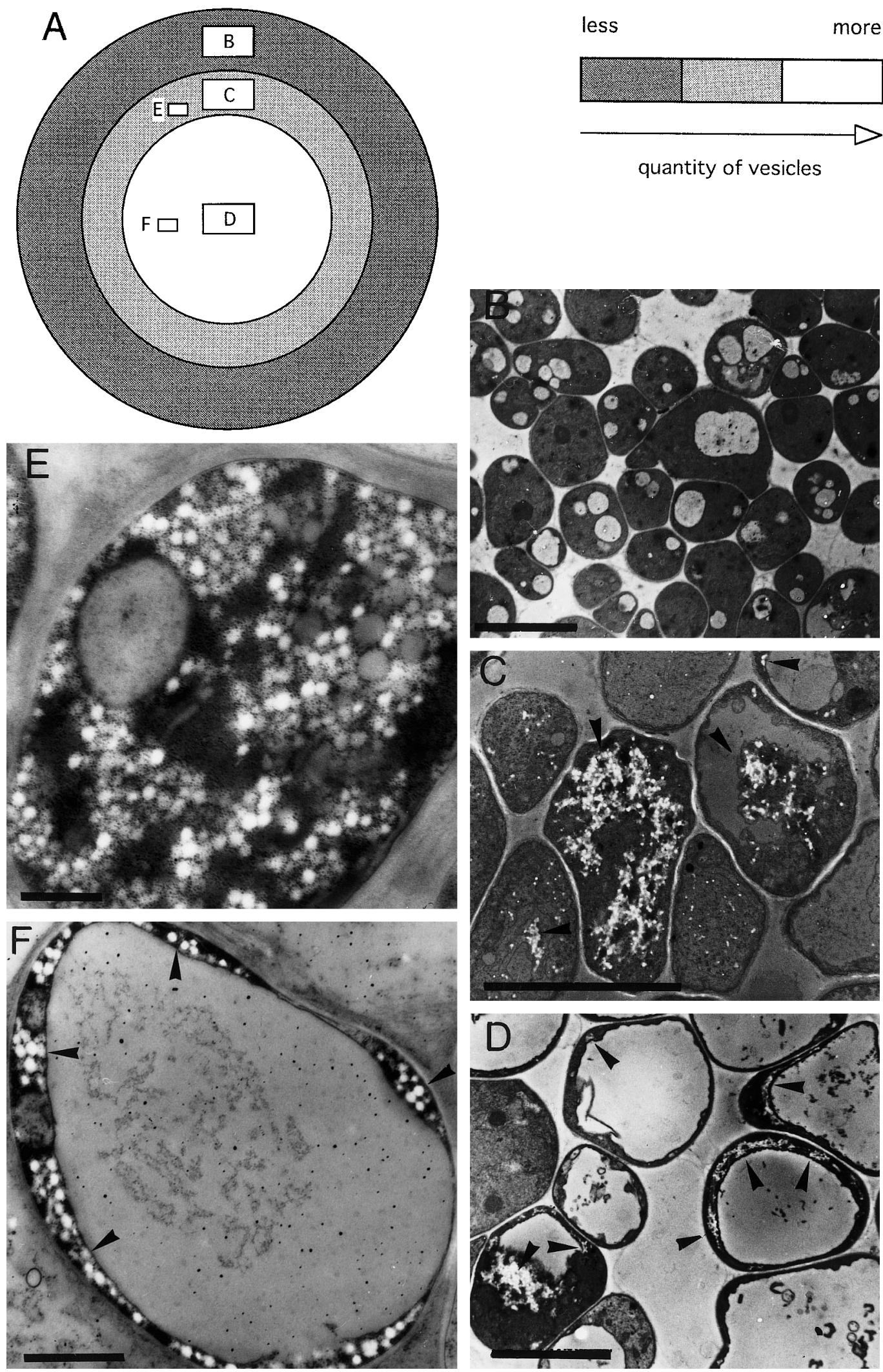

Fig. 4A-F. Transverse sections of the vertically growing transition zone. A Diagrammatic representation of the position of the selective enlargements. The arrowheads indicate microvesicles. B-F Electron micrographs of the peripheral zone (B), the adjacent inner zone $(\mathbf{C}, \mathbf{E})$ and the center (D,F). Ultra-thin sections of embedded specimens (cf. legend to Fig. 3) were cut with a Reichert Ultracut microtome (Reichert, Vienna, Austria), stained with $0.5 \%(\mathrm{w} / \mathrm{v})$ uranylacetate for $15 \mathrm{~min}$ at $45^{\circ} \mathrm{C}$, followed by $0.3 \%(\mathrm{w} / \mathrm{v})$ lead citrate for 1 min at room temperature, and viewed on a Zeiss EM 10 at $60 \mathrm{kV}$. Bars $=5 \mu \mathrm{m}(\mathbf{B}-\mathbf{D}), 1 \mu \mathrm{m}(\mathbf{E}, \mathbf{F})$ 
Table 1. Quantitative analysis of vesicle areas in cross-sections of Flammulina fruiting-body stalks which had been horizontally oriented for $3 \mathrm{~h}$

\begin{tabular}{llll}
\hline & $\begin{array}{l}\text { Total hyphal } \\
\text { area (pixel) }\end{array}$ & $\begin{array}{l}\text { Vesicle area } \\
\text { (pixel) }\end{array}$ & $\begin{array}{l}\text { Vesicle area } \\
(\%)\end{array}$ \\
\hline Upper side hyphae & & & \\
& 175 & 18 & 10.3 \\
& 382 & 12 & 3.1 \\
& 190 & 11 & 5.8 \\
& 498 & 49 & 9.8 \\
& 1664 & 18 & 1.1 \\
Lower side hyphae & & mean $\pm \mathrm{SE}$ & $6.0 \pm 3.6$ \\
& 997 & 274 & 27.5 \\
& 260 & 83 & 31.9 \\
& 205 & 126 & 61.5 \\
& 165 & 70 & 42.4 \\
& 276 & 74 & 26.8 \\
& 610 & 248 & 40.7 \\
& 500 & 155 & 31.0 \\
& & mean $\pm \mathrm{SE}$ & $37.4 \pm 12.2$ \\
\hline
\end{tabular}

bundles if the hyphae could freely move against each other. This situation is modelled by a group of parallel balloons which are held together by two rubber rings at their ends (Figs. 8C,D). However, this model does not mirror the real situation in a stem as the individual hyphae at the periphery are cemented together. If balloons are individually taped as shown in Fig. 8A and glued together at their contact sites, inflation does not cause bending (Figs. 8E,F), except at the right-hand end where gluing was incomplete. The entire bundle behaves like a single non-reinforced control (not shown). Bending can be achieved with bundles of non-reinforced balloons (Fig. 8H), which are glued together and inflated more on the lower side than the upper side (Fig. 8H). This variant models the situation encountered in the fruiting-body stalk. It resembles a bimetal strip which bends after a temperature increase because of different extension coefficients of the two metal layers.

This model still does not explain how the gravistimulus is translated to define the upper and lower flank of a stem, circular in transverse section, which can bend in all directions of space depending on its current orientation relative to the $g$ vector. This applies equally to segments or hyphal bundles taken from any part of the transition zone. Hofmeister (1860) and Banbury (1962, p. 378) concluded that each hypha of the stalk has the potential to gravireact. However, this hypothesis contradicts the model presented above. The problem can be resolved if gravisensing and gravireaction are considered separately. Whereas the current model assigns gravisensing to all hyphae within the transition zone, gravireaction in terms of differential elongation growth occurs only in the hyphal regions at the lower flank.

If the experimental data available for the graviresponse of Flammulina are taken together and fitted into the model presented above, positional information can be conveyed to any spot within the transition zone of the stalk or within a transition zone segment if the following properties are integrated (Fig. 9): (i) Each hypha is
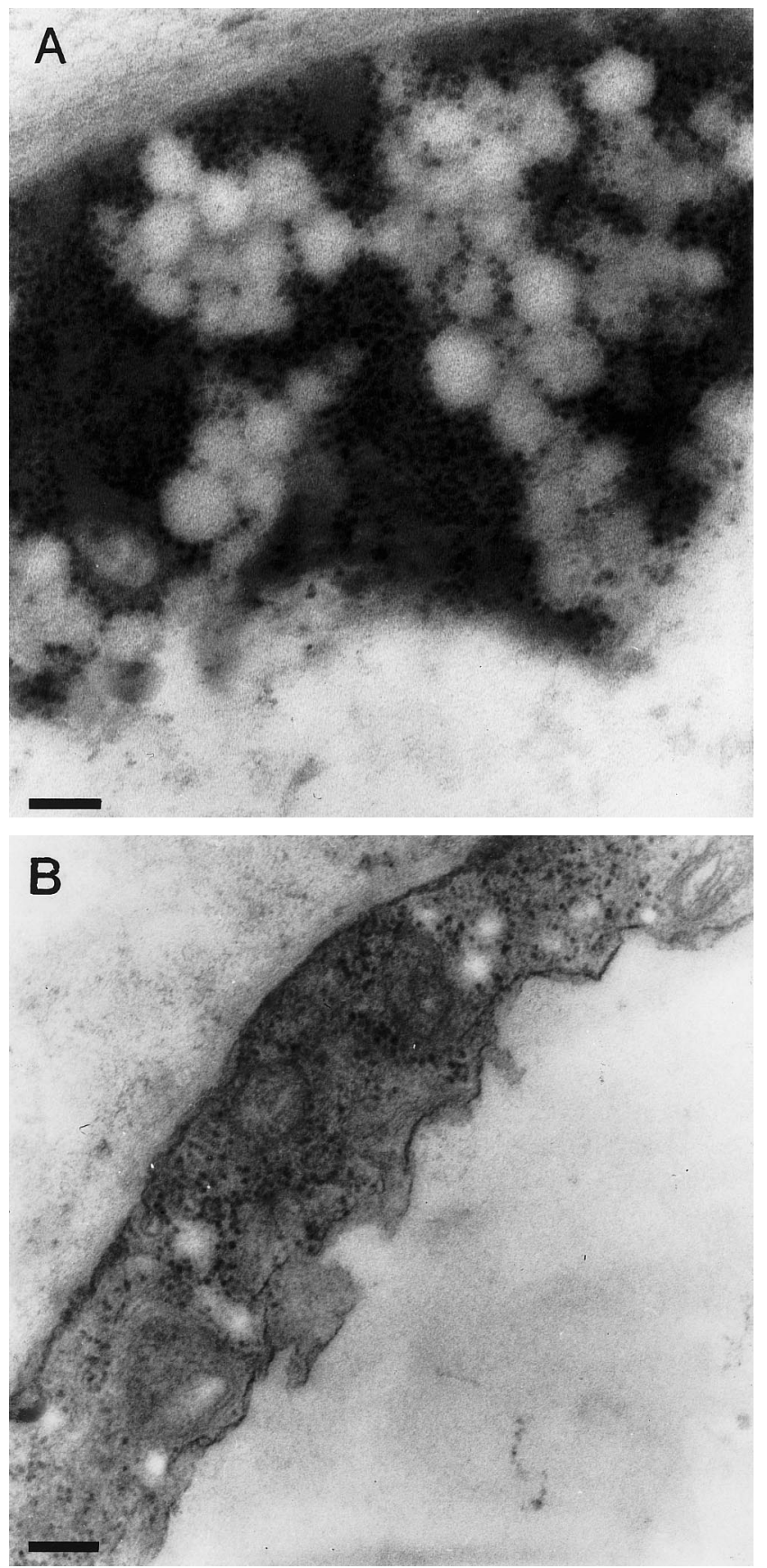

Fig. 5A,B. Microvesicles in growing hyphae of the transition zone of the stalk. A Cytosolic part of a hypha. B Area of transition from the cytosol to a vacuole. High-pressure-freezing technology was applied according to Mendgen et al. (1991). After horizontal reorientation of Flammulina cultures for defined time periods $(0,1,3 \mathrm{~h})$, the fruiting bodies were harvested and infiltrated with $8 \%(\mathrm{v} / \mathrm{v})$ methanol in water under vacuum (30-40 mbar) for 1-4 min. Hand-cut cross-sections, $0.3 \mathrm{~mm}$ thick, of transition zones were high-pressure-frozen at 2300 bar. Control samples reached $-50{ }^{\circ} \mathrm{C}$ within $9 \pm 0.5 \mathrm{~ms}$ (HPM 010; Balzers Union, Liechtenstein). The fungal samples were freezesubstituted at $-90{ }^{\circ} \mathrm{C}$ in anhydrous acetone with $2 \% \mathrm{OsO}_{4}$ for $2 \mathrm{~d}$ and embedded in epon-araldite. Bars $=0.2 \mu \mathrm{m}$

equipped with gravisensing abilities and can detect a dislocation from the vertical position. (ii) This information is used for assigning upper and lower flanks to the 


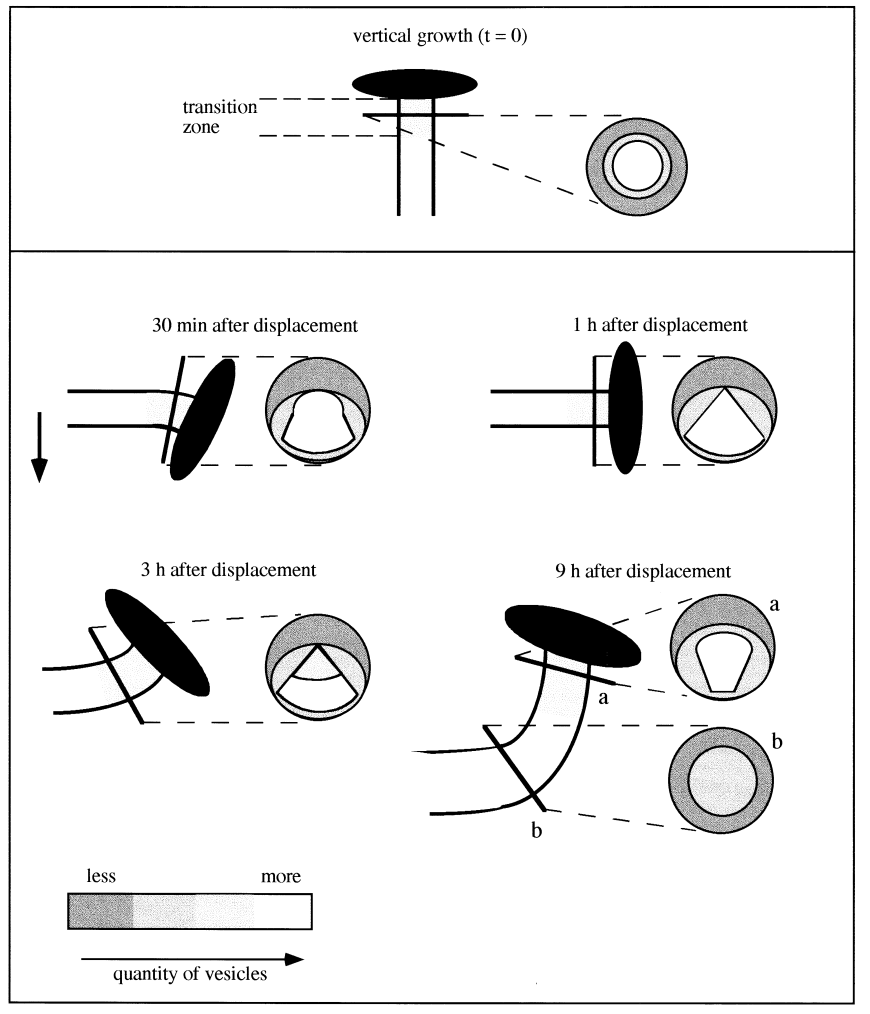

Fig. 6. Diagrammatic representation of the distribution of microvesicles within the transition zone $30 \mathrm{~min}, 1 \mathrm{~h}, 3 \mathrm{~h}$, and $9 \mathrm{~h}$ after the fruiting bodies were placed in a horizontal position. Arrow, gravitational vector

individual hyphae of the transition zone. (iii) Each hypha is also equipped with signalling activities mediating positional information to its surroundings. In this way, a positional signal can be generated and amplified perpendicular to the axis of the transition zone. (iv) A coordinated action of all hyphae together with signal amplification creates a positional gradient, as has been postulated for other systems (e.g. Wolpert 1969). Differential growth is achieved by interpreting positional information, which results in a decrease in growth on the upper side and continued growth on the lower side of a dislocated stem.

It is likely that the nuclei, anchored in a web of actin filaments, are an integral part of the gravisensing mechanism. A change in the gravitational pull is expected to be transmitted to components of the plasma membrane, such as ion channels or membrane receptors, since the actin filaments are attached to this membrane system (Monzer 1995). Incidently, the number of nuclei is greater within the stem and within parts of the cap than in the dikaryotic mycelial hyphae. Other candidates for statoliths, which are heavy enough to be pulled by acceleration forces of $1 \mathrm{~g}$ to an appreciable extent, have not been detected, nor has gravity-related flotation of less-dense vacuoles been verified in this system. Monzer (1995) presented evidence for the involvement of actin filaments in graviperception of Flammulina. The actinfilament-disrupting antibiotic cytochalasin D suppresses
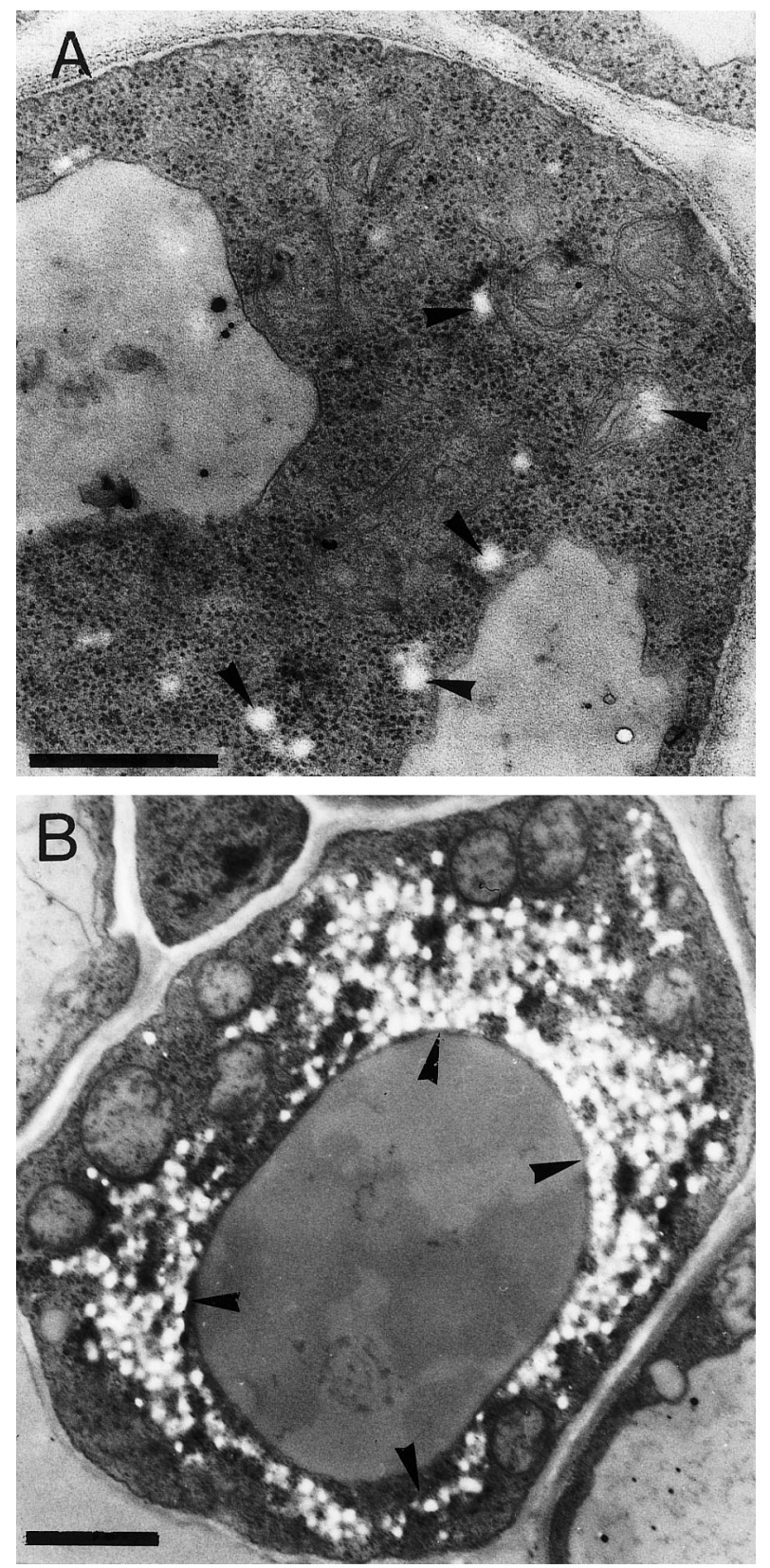

Fig. 7A,B. Microvesicles of the transition zone on the upper side (A) and the lower side (B) of the stalk $1 \mathrm{~h}$ after the fruiting bodies were placed in a horizontal position. The arrowheads indicate microvesicles. Bars $=0.5 \mu \mathrm{m}(\mathbf{A})$ and $1 \mu \mathrm{m}(\mathbf{B})$

gravitropic curvature whereas the microtubulin inhibitor oryzalin does not interfere with this process.

Positional information can be transmitted to the opposite flanks of a hyphal system if each hypha is equipped not only with gravisensing abilities but also with signalling capabilities. The simplest possibility would be an export of cytosolic growth factor at the lower side of each hypha and an import at its upper side from the surroundings. Since the presently known plant hormones (including methyljasmonate) do not influence the growth of fruiting-body stems, another substance may be involved. Thereby, a gradient could be formed 

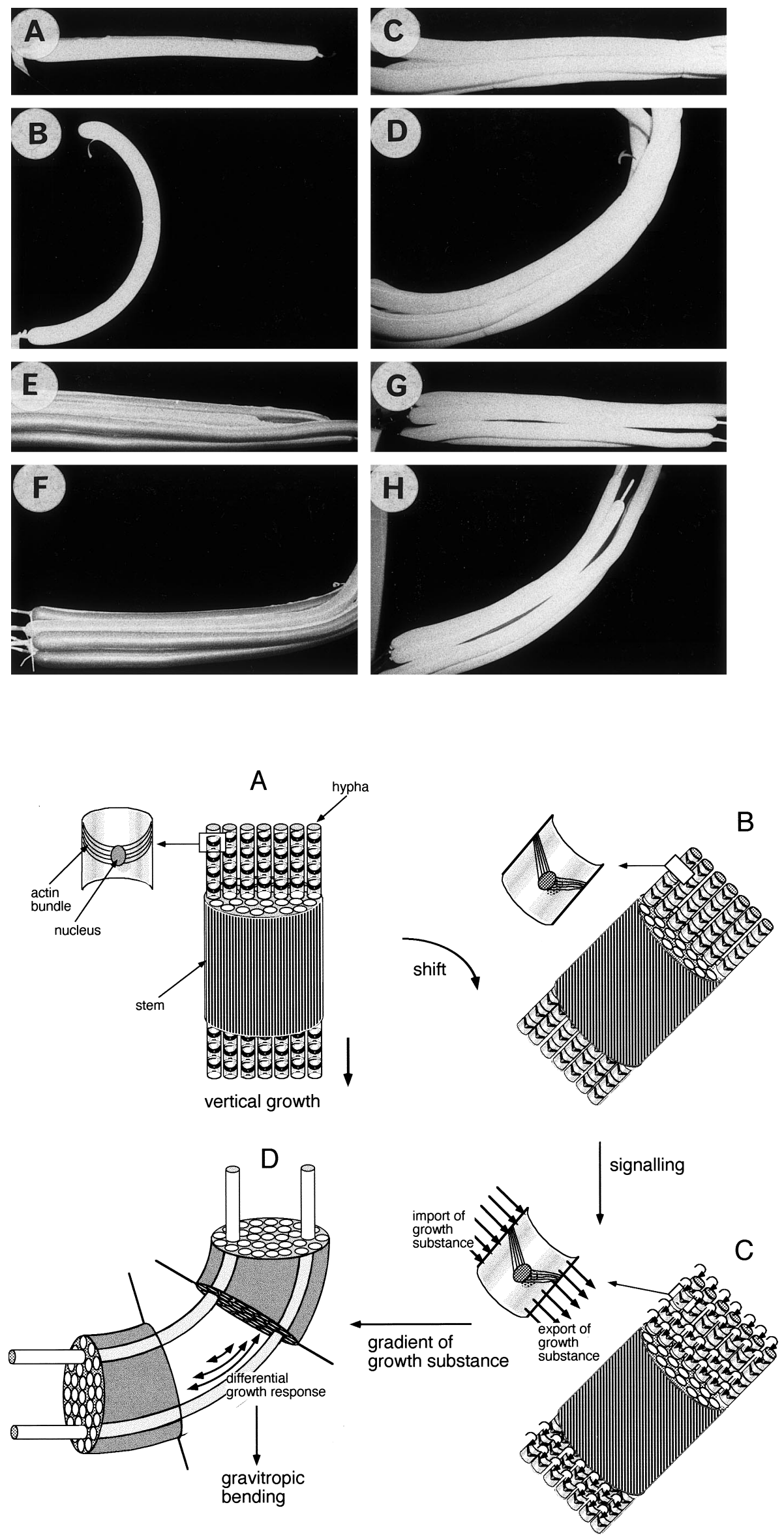

Fig. 8A-H. Balloon model representing bending of hyphae and hyphal bundles in the stage of low inflation $(\mathbf{A}, \mathbf{C}, \mathbf{E}, \mathbf{G})$, and high inflation $(\mathbf{B}, \mathbf{D}, \mathbf{F}, \mathbf{H})$ Single balloons were reinforced on their prospective upper side with adhesive tape (A-F) or left without adhesive tape $(\mathbf{G}, \mathbf{H})$. Bundles are held together with two rubber rings $(\mathbf{C}, \mathbf{D})$ or with glue (Fixogum; Marabu) connecting the contact sites $(\mathbf{E}-\mathbf{H})$

Fig. 9A-C. Diagrammatic representation of the proposed events in the gravitropism of Flammulina. A Each hypha is equipped with individual gravisensors. This property is attributed to the nuclei, which are suspended in a web of actin filaments anchored in the plasma membrane. Arrow, gravitional vector. B Any shift from the vertical position is converted into a change in the gravitational pull exerted on the plasma membrane. This leads to a functional distinction of upper and lower flanks of the individual hyphae within the transition zone. C Each hypha is also equipped with signalling capabilities, e.g. by importing a growth factor at its upper flank and secreting it at its lower flank. In this way a positional signal can be generated and amplified perpendicular to the axis of the transition zone by the coordinated action of all hyphae. D This gradient is then translated into a differential growth response leading to gravitropic bending 
with a concentration increase from the upper to the lower flank. This gradient is assumed to trigger vesicle formation in a concentration-dependent manner.

The existence of a growth-promoting factor regulating the elongation of agaric stipes has been postulated by several authors (e.g. Hagimoto and Konishi 1959; Gruen 1979). It is thought to be synthesized by the lamellae of the cap and transported to the stalk. However, it is not likely that this growth factor is identical to the signal which moves in response to a gravitropic stimulus from the upper to the lower flank (perpendicular to the axis) of a tilted stem. Indirect evidence is available that the latter is a water-soluble, transmissible factor. If stem segments of Flammulina are kept in a horizontal position under water, they do not bend but do elongate, whereas controls kept in silicone oil are gravitropic (Haindl and Monzer 1994). This experimental separation of gravitropic curvature and elongation growth indicates the involvement of two separate regulatory mechanisms.

A joint action of gravisensing by the individual hyphae of the transition zone and signalling perpendicular to the axis of the stem not only explains gravitropic bending of intact stalks but also of small segments irrespective of their original location within the stalk as long as there are sufficient cells to create a positional gradient. This process also explains paradoxical responses of the stem. If an isolated fruiting body is immobilized at the cap in a horizontal position, the stem shows negative gravitropism followed by an overshoot (Moore et al. 1996). This response can be explained by the fact that the transition zone cannnot leave its horizontal position. As the lower side of the stem is continuously promoted in growth, the free end of the stem is forced to an upward movement and continues bending.

Although positional information could also be conveyed to the flanks by a concentration gradient of a growth inhibitor enriched on the upper side, the involvement of a growth-promoting factor appears to be more likely because of the fast accumulation of microvesicles on the lower side preceding the elongation response of this region.

As cell elongation depends upon an increase in cell volume, which is due to water uptake, the limiting factors have to be considered. The rate of volume increase depends on the extensibility of the cell wall and the turgor pressure exceeding a threshold below which no plastic extensibility can occur. It can be inferred from Figs. 5 and 7 that cell extension is related to microvesiculation which contributes to vacuolation. It is assumed that microvesicles deliver a highly concentrated solution that drives osmotic water intake. A regulation exclusively by an increase in plastic extensibility does not fit into these observations. On the other hand, it is not clear how wall stretching is regulated. Micrographs (Kern and Hock 1994, Fig. 18) show apparent fusion of Golgi vesicles with the plasma membrane, but no differences in frequency of vesicle fusion were subsequently observed between upper and lower sides of horizontally oriented hyphae. This underlines the finding that a major subcellular event involved in gravitropic bending is the accumulation of microvesicles in the lower part of the stem.

\section{Conclusion}

Flammulina provides an excellent model for studying gravitropism of fruiting-body stems. In this system, graviperception and gravireaction are restricted to a small part of the apical zone. Subcellular changes in response to a gravistimulus are already observed after $30 \mathrm{~min}$. The key components hypothesized to be involved in the gravitropic response are summarized in Fig. 9. Although several details must remain speculative at this time, the overall concept, i.e. gravisensing ability of all hyphae of the transition zone and generation of positional information within the stem by signalling in the axial direction, is compatible with experimental data and represents the minimal requirements for a cooperative, highly ordered behavior of a hyphal system.

This work was supported by a grant of the Deutsche Agentur für Raumfahrtangelegenheiten (DARA, Bonn) to B.H. We thank Ms. Stefanie Rauchalles and Dr. Alexander Hahn for their assistance with the preparation and photography of the balloon model. Mrs. Ute Labitzke (Department of Phytopathology, University of Konstanz), and Dr. Hans C. Bartscherer (Department of Physics of the TU München at Freising-Weihenstephan), helped with the TEM.

\section{References}

Aylor DE, Parlange JY, Krikorian AD (1973) Stomatal mechanics. Am J Bot 60: 163-171

Banbury GH (1962) Gravitropism of lower plants. In: Ruhland W (ed) Handbuch der Pflanzenphysiologie, vol 17.2. Springer, Berlin, pp 344-377

Buller AHR (1909) Researches on fungi, vol 1. Longman, Green and Co., London

Dennison DS (1961) Tropic responses of Phycomyces sporangiophores to gravitational and centrifugal stimuli. J Gen Physiol 45: 23-38

Dennison DS, Shropshire W (1984) The gravireceptor of Phycomyces: its development following gravity exposure. J Gen Physiol 84: 845-859

Fultz SA (1988) Fruiting at high temperature and its genetic control in the basidiomycete Flammulina velutipes. Appl Environ Microbiol 54: 2460-2463

Greening JP, Holden J, Moore D (1993) Distribution of mechanical stress is not involved in regulating stem gravitropism in Coprinus cereus. Mycol Res 97: 1001-1004

Gruen HE (1979) Control of rapid stipe elongation by the lamellae in fruit bodies of Flammulina velutipes. Can J Bot 57: 1131-1135

Haberlandt G (1900) Über die Perzeption des geotropischen Reizes. Ber Dtsch Bot Ges 18: 261-272

Hagimoto H, Konishi M (1959) Studies on the growth of fruit body of fungi. I: Existence of a hormone active to the growth of fruit body in Agaricus bisporus (Lange) Sing. Bot Mag Tokyo 72: 353-366

Haindl E, Monzer J (1994) Elongation growth and gravitropic curvature in the Flammulina velutipes (Agaricales) fruiting body. Exp Mycol 18: 150-158

Hofmeister W (1860) Über die durch die Schwerkraft bestimmten Richtungen von Pflanzentheilen. Ber Königl Sächs Gesellschaft der Wissenschaften zu Leipzig. Math.-phys. Classe 12: $175-213$ 
Hofmeister W (1863) Ueber die durch die Schwerkraft bestimmten Richtungen von Pflanzentheilen. Jahrb Wiss Bot 3: 77-114

Kern VD, Hock B (1994) Gravitropism of fungi - Experiments in space. In: Proc 5th Eur Symp on Life Sci Res in Space, Arcachon, France, 1993, pp 49-60

Knoll F (1909) Untersuchungen über Längenwachstum und Geotropismus der Fruchtkörperstiele von Coprinus stiriacus. Sitzungsber Kaiserl Akad Wiss Wien, Mathem-Naturwiss Klasse Abt 1 118: 575-634

Kraus G (1867) Die Gewebespannung des Stammes und ihre Folgen. Bot Z 25: 105-142

Kutschera U (1992) The role of the epidermis in the control of elongation growth in stems and coleoptiles. Bot Acta 105: 246-252

Mendgen K, Welter K, Scheffold F, Knauf-Beiter G (1991) High pressure freezing of rust infected plant leaves. In: Mendgen K, Lesemann D-E (eds) Electron microscopy of plant pathogens. Springer, Heidelberg, pp 31-42

Monzer J (1995) Actin filaments are involved in cellular graviperception of the basidiomycete Flammulina velutipes. Eur J Cell Biol 66: 151-156

Monzer J, Haindl E (1994) Evidence for the involvement of the cytoskeleton and organelles in graviperception of the basidiomycete Flammulina velutipes. In: Proc 5th Eur Symp on Life Sci Res in Space, Arcachon, France, 1993. ESA Publ. Division, Noordwijk, ESA SP-366 pp 89-93

Monzer J, Haindl E, Kern V, Dressel K (1994) Gravitropism of the basidiomycete Flammulina velutipes: morphological and physiological aspects of the graviresponse. Exp Mycol 18: 7-19

Moore D (1991) Perception and response to gravity in higher fungi - a critical appraisal. New Phytol 117: 3-23

Moore D, Hock B, Greening JP, Kern VD, Frazer LN, Monzer J (1996) Gravimorphogenesis in agarics. Mycol Res 100: 257-273

Němec B (1900) Über die Wahrnehmung des Schwerkraftreizes bei den Pflanzen. Ber Dtsch Bot Ges 18: 241-245

Sachs J (1865) Schwerkraft. Handbuch der Experimentalphysiologie der Pflanzen, Engelmann, Leipzig, pp 88-112
Sachs J (1879) Über Ausschließung der geotropischen und heliotropischen Krümmungen während des Wachsens. Arb Bot Inst Würzburg 2: 209-225

Sievers A, Buchen B, Volkmann D, Hejnowicz Z (1991) Role of the cytoskeleton in gravity perception. In: Lloyd CW (ed) The cytoskeletal basis of plant growth and form. Academic Press, London, pp 169-182

Schmitz J (1842) Mycologische Beobachtungen, als Beiträge zur Lebens- und Entwicklungsgeschichte einiger Schwämme aus der Klasse der Gastromyceten und Hymenomyceten. Linnaea 16: $168-200$

Spurr AR (1969) A low-viscosity epoxy resin embedding medium for electron microscopy. J Ultrastruct Res 26: 31-43

Wessels JGH (1993) Wall growth, protein excretion and morphogenesis in fungi. New Phytol 123: 397-413

Wolpert L (1969) Positional information and the spatial pattern of cellular differentiation. J Theor Biol 25: 1-47

\section{Additional references (this issue)}

Baluška F, Hasenstein KH (1997) Root cytoskeleton: its role in perception of and response to gravity. Planta 203: S69-S78

Braun M (1997) Gravitropism in tip-growing cells. Planta 203: S11S19

Perbal G, Driss-Ecole D, Tewinkel M, Volkmann D (1997) Statocyte polarity and gravisensitivity in seedling roots grown in microgravity. Planta 203: S57-S62

Ruyters G, Scott TK (1997) Future research in plant biology in space: summary of critical issues and recommendations of the workshop. Planta 203: S211-S213

Sack FD (1997) Plastids and gravitropic sensing. Planta 203: S63S68

Scherer GFE (1997) General discussion on graviperception. Planta 203: S107-S111 